\title{
Analysis of Antioxidant and Antimicrobial Properties of Different Plant Species Growing in Shar Mountains
}

\author{
Gulnur Arabaci,", Busra Tosun ${ }^{1}$, Cengiz Cesko ${ }^{2}$ \\ ${ }^{1}$ Department of Chemistry, Faculty of Art and Science, Sakarya University, TR-54050, Sakarya, Turkey \\ ${ }^{2}$ Department of Food Science and Biotechnology, University Business and Technology - UBT Lagjja Kalabria p.n., 10000 Pristina, \\ Republic of Kosovo
}

Received January 11, 2021; Revised March 1, 2021; Accepted March 23, 2021

\section{Cite This Paper in the following Citation Styles}

(a): [1] Gulnur Arabaci, Busra Tosun, Cengiz Cesko, "Analysis of Antioxidant and Antimicrobial Properties of Different Plant Species Growing in Shar Mountains," Food Science and Technology, Vol. 9, No. 1, pp. 1 - 6, 2021. DOI: 10.13189/fst.2021.090101.

(b): Gulnur Arabaci, Busra Tosun, Cengiz Cesko (2021). Analysis of Antioxidant and Antimicrobial Properties of Different Plant Species Growing in Shar Mountains. Food Science and Technology, 9(1), 1 - 6. DOI: 10.13189/fst.2021.090101.

Copyright $\bigcirc 2021$ by authors, all rights reserved. Authors agree that this article remains permanently open access under the terms of the Creative Commons Attribution License 4.0 International License

\begin{abstract}
The goal of the present work was to analyze the antioxidant and antimicrobial properties of methanol extracts of different plant species (Datura stramonium seed, Datura stramonium leaf, Verbascum thapsus, Rosmarinus officinalis and Thymus vulgaris) grown in Shar Mountains, Kosovo. Three different methods were utilized to analyze the antioxidant activities of the plant extracts and their antimicrobial activities were determined by using agar well diffusion method. Considering the antioxidant results, Thymus vulgaris extract had the highest DPPH (2,2-diphenyl-1-picrylhydrazyl) radical scavenging activity with the IC50 value of $6271.43 \pm 0.03 \mathrm{mg} / \mathrm{L}$. The chelating property of iron ions was observed with the highest activity in Thymus vulgaris, Verbascum thapsus and Datura stramonium leaves and they showed the highest reducing power activity. In addition, the antimicrobial activities of the plant species were investigated against Escherichia coli (ATCC 25922), Staphylococcus aureus (ATCC 29213), Bacillus cereus (SBT8), Bacillus subtilis (ATCC 6633) and Bacillus subtilis (ATCC 6051) by using well diffusion method. The results demonstrated that all plant samples had antibacterial effects on all bacteria tested. However, among the extracts, Rosmarinus officinalis and Thymus vulgaris showed the highest antibacterial activity against bacteria.
\end{abstract}

Keywords Antimicrobial, Antioxidant, Rosmarinus officinalis, Thymus vulgaris, Datura stramonium, Verbascum thapsus

\section{Introduction}

For centuries, people have been using plants for food as well as for treatment purposes. Traditional medicines obtained from plant extracts are used more favourably than synthetic agents in many fields such as medicine, pharmaceuticals, food processes and cosmetics due to their therapeutic effects, rich contents and less toxic $[1,2,3]$. Successful extraction of biologically active molecules such as antioxidants and antimicrobials from plants is important for their applications. For this purpose, various solvents such as ethanol, methanol and diethyl ether are commonly used.

The oxidant-antioxidant balance of the organism is important for health and a healthy life. Reactive Oxygen Species (ROS) formed during various metabolic reactions can cause significant oxidative damage to lipids, proteins and DNA, resulting in impairment of cell functions. The inadequate relationship between antioxidant defences and ROS formation causes oxidative stress in living systems. Oxidative stress in organisms is caused by an increase in free radical production or a deficiency in the antioxidant defence system. Reactive oxygen species are dangerous for living systems by adversely oxidizing the biomolecules that can lead to degenerative processes such as tissue damage, cell death or aging, cancer and skin diseases in living systems [4,5]. Antioxidants reduce these free radicals, which pose a threat to cells, and convert them into less harmful products [6]. Although there are 
various natural plant-derived and synthetic antioxidants used in some industries such as food, cosmetic and pharmaceutical, new natural antioxidants still need to be found with high antioxidant activities.

For many years, among the public, plants have been used as an antimicrobial agent for killing bacteria as well as many other uses such as food, medicine and antioxidant. Antimicrobial compounds of plants are usually found in their essential oil parts, responsible for the characteristic aroma of the plant [7]. Since microorganisms generally develop resistance to many antibiotics, it is always necessary to find new natural plant-based antimicrobial agents [8].

In the current work, we aimed to determine the antioxidant and antimicrobial properties of different plant species such as Datura stramonium leaf, Datura stramonium seed, Rosmarinus officinalis, Thymus vulgaris, Verbascum Thapsus grown in the Shar Mountains of Kosovo. These plants are generally used in the cure of several diseases as a public medicine among the public living in Kosovo. Rosmarinus officinalis and Thymus vulgaris plants in general are known good antioxidant and antimicrobial agents however, within our awareness; the tested plants from Kosovo have not yet been studied as antioxidants and antibacterial agents. Therefore, this study is the first to analyse the antioxidant and antimicrobial properties of these plants.

\section{Materials and Methods}

\subsection{Extraction of Plants}

Plant samples were collected from the Shar Mountains of Kosovo and dried. Dried plant samples were stored in sterile glass jars at room temperature in a dark environment without direct sunlight until the study started. Extracts in methanol were prepared for each plant species. Subsequently, methanol was removed by a rotary evaporator to yield phenolic extract.

\subsection{Determination of DPPH Free Radical Scavenging Activity}

The method was modified and used in accordance with the method of Blois work [9]. Trolox and BHT (butylated hydroxytoluene) were used as standards. 2,2-diphenyl-1-picrylhydrazyl (DPPH) solution was added on samples at concentrations ranging from $100 \mu \mathrm{g}$ to $500 \mu \mathrm{g}$ in $4 \mathrm{~mL}$ volume. Methanol was used as blank. The mixture was kept for $30 \mathrm{~min}$ at room temperature and their absorbance results were observed at $517 \mathrm{~nm}$. The absorbance values of the samples were evaluated against the control. The scavenging activity was estimated by using the subsequent formula:

$$
\% \text { inhibition }=\frac{A b-A a}{A b} \times 100
$$

in which $A a$ is absorption of the extract and $A b$ is absorption of the blank sample.

$\mathrm{IC}_{50}$ (50\% inhibition of the extract concentration) was calculated from the plot of extract concentration against inhibition percentage.

\subsection{Determination of Chelating Activity of Iron (II) Ions}

$3.7 \mathrm{~mL}$ of pure water and $100 \mu \mathrm{L}$ of $2 \mathrm{mM}$ of $\mathrm{FeCl}_{2}$ solution were mixed with $1 \mathrm{~mL}$ the sample and incubated for 30 minutes. Then, $5 \mathrm{mM}$ ferrozine solution was mixed to the sample mixture and mixed. 10 minutes later, the reaction absorbance values were obtained at $562 \mathrm{~nm}$ and control was only pure water. EDTA was preferred as standard at concentrations of $10-1000 \mu \mathrm{g} / \mathrm{mL}$. The percent inhibition of ferrozine- $\mathrm{Fe}^{+2}$ complex was calculated based on the following formula:

$\%$ Chelating Activity $\left.=\left[\mathrm{A}_{\text {Comtrol}}-\mathrm{A}_{\text {Sample }}\right) / \mathrm{A}_{\text {control }}\right] \times 100$

Where $A_{\text {Control }}$ was the absorbance of control at $562 \mathrm{~nm}$ and $\mathrm{A}_{\text {Sample }}$ was the absorbance of the sample at $562 \mathrm{~nm}$.

\subsection{Determination of Reducing Power}

Plant extracts were prepared in various concentrations $(5-100 \mu \mathrm{g} / \mathrm{mL})$ and mixed with standard substance in 1 $\mathrm{mL}$ of solution, $2.5 \mathrm{~mL}$ of phosphate buffer $(0.2 \mathrm{M}$, $\mathrm{pH}=6.6)$ and $2.5 \mathrm{~mL}$ of $1 \% \mathrm{~K}_{4} \mathrm{Fe}(\mathrm{CN})_{6} \cdot 3 \mathrm{H}_{2} \mathrm{O}$. After the mixtures were incubated at $50^{\circ} \mathrm{C}$ for 20 minutes, $2.5 \mathrm{~mL}$ of $10 \%$ TCA was put into the mixture and centrifuged at $2500 \mathrm{rpm}$ for ten minutes. After centrifugation, $2.5 \mathrm{~mL}$ of distilled water was taken from the supernatants and $0.5 \mathrm{~mL}$ of $0.1 \% \mathrm{FeCl}_{3}$ solution. Absorbance at $700 \mathrm{~nm}$ was read. Water was blank. BHT and ascorbic acid were utilized as commercial antioxidant standards.

\subsection{Determination of Antimicrobial Effect by Well Diffusion Method}

In this work, it had been also investigated the antimicrobial activity of the plant species against Escherichia coli (ATCC 25922), Staphylococcus aureus ATCC 29213), Bacillus cereus (SBT8), Bacillus subtilis (ATCC 6051) and Bacillus subtilis (ATCC 6633) by using well diffusion method according to Magaldi et al. [10].

First, the plant extracts in methanol were dissolved in methanol. Then, the medium prepared by mixing Mueller Hinton Agar with $1000 \mathrm{ml}$ distilled water was transferred to petri dishes and the petri dish containing the solidified media containers in the refrigerator at $+4^{\circ} \mathrm{C}$ until use. 6 $\mathrm{mm}$ diameter wells were formed for the samples and then $40 \mu \mathrm{L}(20 \mu \mathrm{L}+20 \mu \mathrm{L})$ extracts were added to the wells in petri dishes. As negative control, $40 \mu \mathrm{L}$ methanol was used. $200 \mu \mathrm{L}$ (containing $10^{6}$ colonies according to Mc Farland 0.5 equality) from the culture suspension of 
microorganisms used in the test were transferred to petri dishes containing Mueller Hinton Agar and spread on the surface with the swab. The dishes were kept about 24 hours at $37^{\circ} \mathrm{C}$. The antimicrobial effect around the wells in which the extracts were added was determined by measuring the diameter of the zones.

\subsection{Statistical Analysis}

All the antioxidant experiments were repeated in the same way three times. The evaluated results were presented as mean values and standard deviation (SD).

\section{Results}

Identification of bioactive molecules from plant extracts is very important for various applications such as antioxidant and antimicrobial agents. New studies to find new resources are increasing day by day. In this study, antioxidant properties of different plants grown in Shar Mountains of Kosovo were determined. These plants were Datura stramonium leaf, Datura stramonium seed, Verbascum thapsus (Verbascum), Rosmarinus officinalis (Rosemary) and Thymus vulgaris (Thyme).

For this purpose, the dried plants were extracted bye methanol first. Three of the commonly used antioxidant methods were then applied to the extracts to determine their antioxidant properties. The methods used in the study were DPPH radical scavenging activity, ferrous ion chelating activity and reducing power methods.

DPPH radical scavenging activity method has been used to analyse the antioxidant activity of the plant extracts. This method is considered that the determination of scavenging activity of antioxidants is a valid and easy assay since the radical substance is stable and does not need to be produced as required in other radical scavenger experiments [11].

The scavenging activities on DPPH radicals by the plant extracts are given in Figure 1. Trolox and BHT were used as common standard antioxidants. Among the extracts, maximum radical scavenging effect was the $\mathrm{IC}_{50}$ value of $6271.43 \pm 0.03 \mathrm{mg} / \mathrm{L}$ for the thyme methanol extract. However, all tested extracts had very good DDPH radical scavenging activities at a concentration of 100 $\mathrm{mg} / \mathrm{L}$. Even all plant extracts tested had DPPH radical scavenging activity much better than the synthetic standard BHT. In this study, it was found that thyme extract showed much better activity than rosemary, verbascum, Datura stramonium leaf and seed extracts.

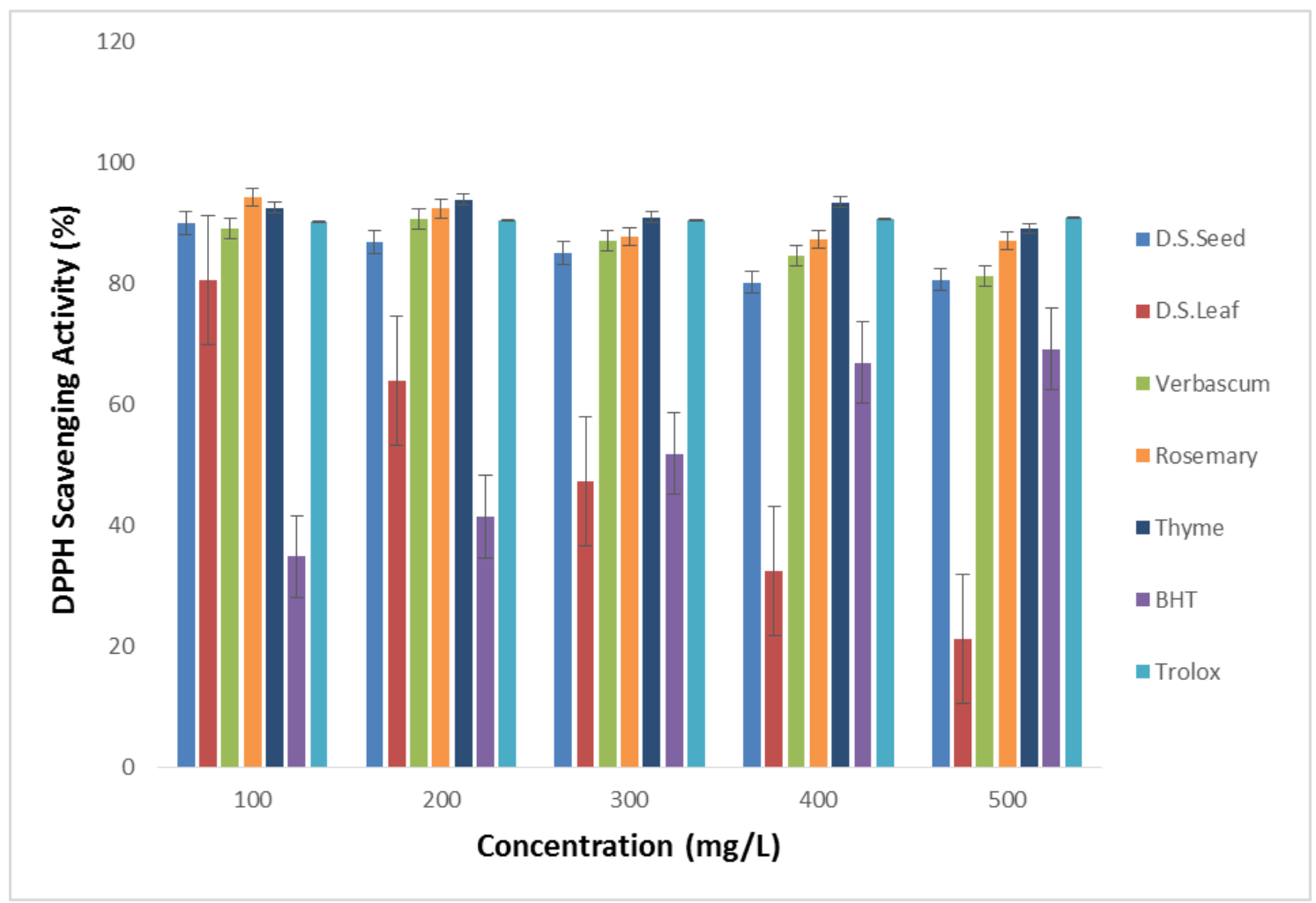

Figure 1. Free radical scavenging ability of compounds on DPPH radicals (\%). Vertical bars represent SD 
Another method for determining antioxidant capacity depends on the ability of certain antioxidant compounds to chelate metal ions (particularly iron and copper). Iron atom produces the free radicals during the Fenton and Haber-Weiss reaction. The chelating of metals forms stable complexes that retain metals and prevent their participation in the formation of free radicals [12]. The chelating activity of iron (II) ions were performed with ferrozine. Ferrozine gives a colored complex with iron (II) atom which can be monitored at $562 \mathrm{~nm}$. In the existence of the antioxidant compound, the formation of complex with ferrozine is negatively affected and the complex formation can be reduced. In this work, methanol extracts of the plant species and EDTA as a standard were examined and the results were presented in Figure 2. All extracts tested had ferrous ion chelating activity, however, thyme and Verbascum extracts had the best chelating activity with the same activity as standard EDTA. The order of metal chelating activities of extracts is as follows; thyme > verbascum> rosemary > Datura stramonium leaf $>$ Datura stramonium seed (Figure 2).

Reducing power activity was another way to assess antioxidant activity. This method is based on compounds which have the reduction potential. These compounds react with potassium ferricyanide $\left(\mathrm{Fe}^{3+}\right)$ to generate potassium ferrocyanide $\left(\mathrm{Fe}^{2+}\right)$. The product then reacts with iron chloride to form the iron-iron complex with maximum absorption at $700 \mathrm{~nm}$ [13]. In this study, methanol extracts of the plants tested and BHT and ascorbic acid as antioxidant standards were evaluated and the results were shown in Figure 3. According to the results obtained, all extracts have close reducing power, but have lower values than the standards. The order of reducing power activities of the plant extracts is Datura stramonium leaf > rosemary > verbascum > Datura stramonium seed $>$ thyme.

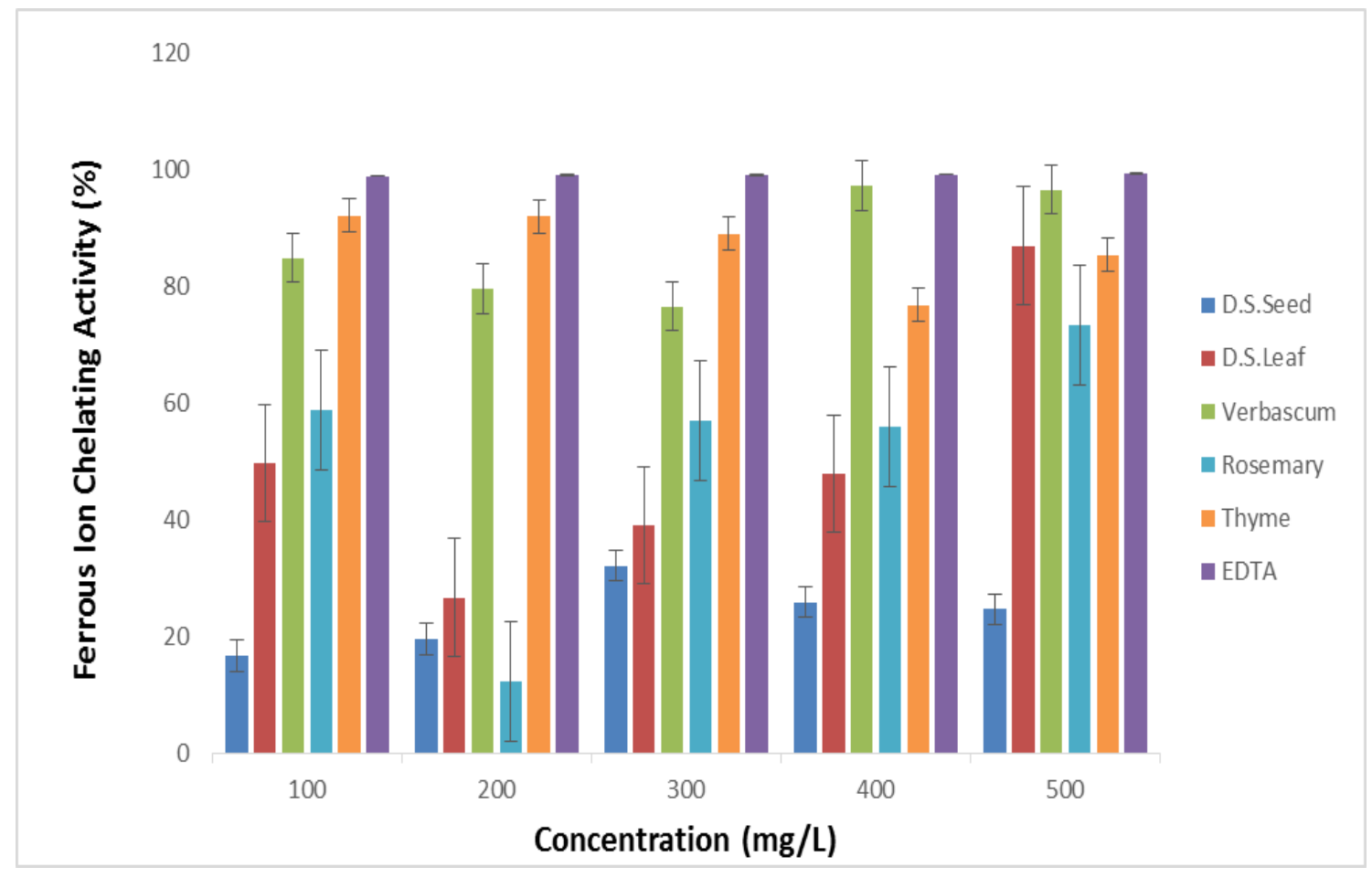

Figure 2. Demonstration of the ability of plant extracts to chelate iron ions 


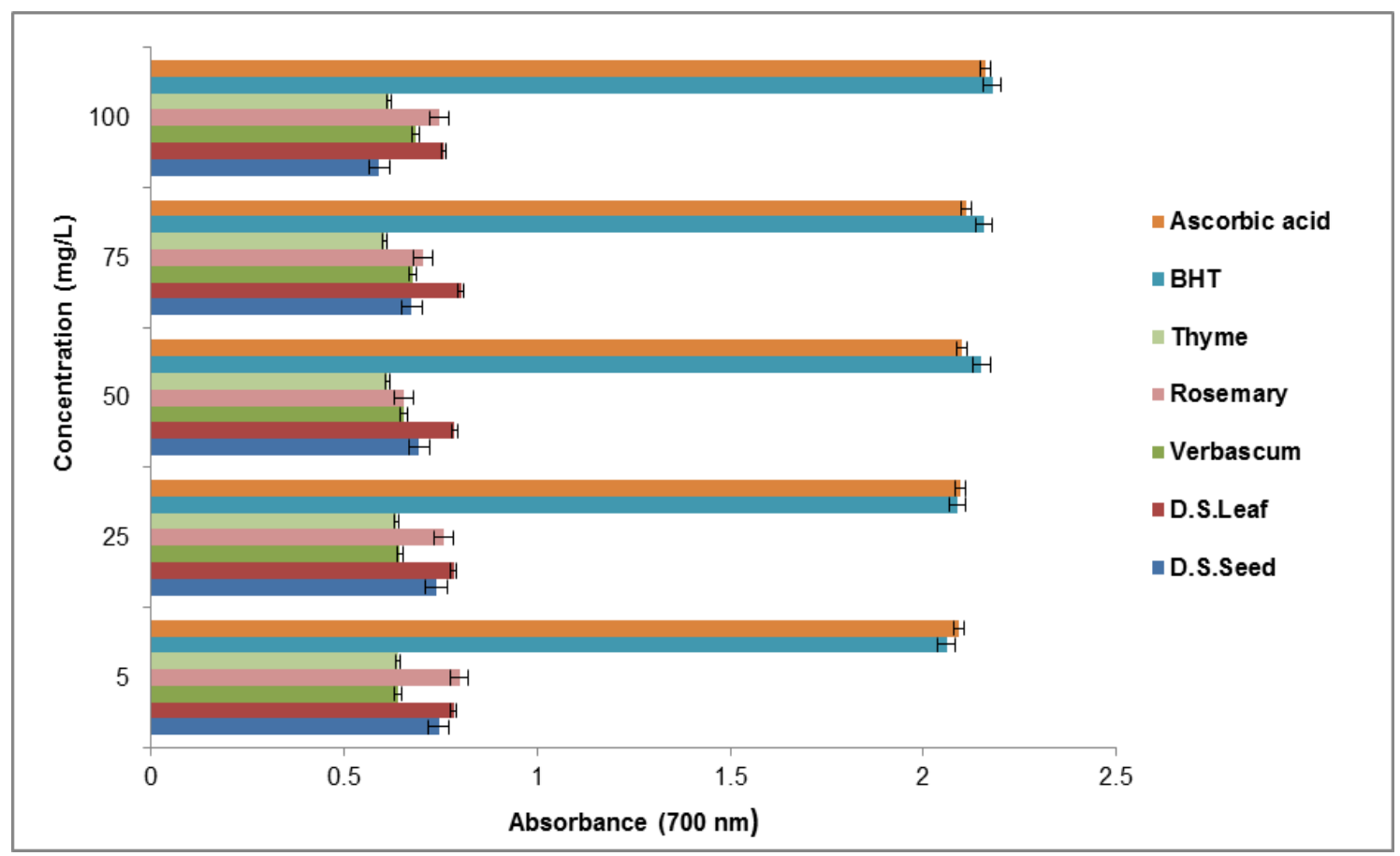

Figure 3. Reduction of the power test absorbance varies at $700 \mathrm{~nm}$ in the existence of various concentrations of plant extracts. Vertical bars represent SD

Table 1. Antimicrobial activity of plant extracts and standard antibiotic

\begin{tabular}{|c|c|c|c|c|c|c|c|}
\hline \multirow{2}{*}{ Bacteria } & \multicolumn{7}{|c|}{ Plant extracts and Standard Antibiotic* } \\
\cline { 2 - 9 } & 1 & 2 & 3 & 4 & 5 & AMP & CTX \\
\hline Escherichia coli (ATCC 25922) & 11 & 8 & 8 & 9 & 8 & 20 & 32 \\
\hline Staphylococcus aureus (ATCC 29213) & 12 & 8 & 10 & 22 & 12 & 21 & 20 \\
\hline Bacillus cereus (SBT8) & 10 & 16 & 15 & 21 & 12 & 11 & 24 \\
\hline Bacillus subtilis (ATCC 6051) & 10 & 11 & 8 & 25 & 15 & 40 & 30 \\
\hline Bacillus subtilis (ATCC 6633) & - & 12 & - & 20 & 9 & 38 & 30 \\
\hline
\end{tabular}

*Inhibition zone diameter in millimeters.

$(\mathrm{AMP}=$ Ampicillin $(10 \mu \mathrm{g}), \mathrm{CTX}=$ Cefotaxime $(30 \mu \mathrm{g}), 1$ : Datura stramonium Seed, 2: Datura stramonium leaf, 3: Verbascum thapsus, 4: Rosmarinus officinalis 5: Thymus vulgaris.)

The antibacterial activity of methanol extracts and standard antibiotics (AMP and CRX) against 5 different bacteria was examined by the presence and absence of inhibition zones using well-diffusion method. In the present work, Gram-positive (Staphylococcus aureus, Bacillus subtilis (ATCC 6051) and Bacillus subtilis (ATCC 6633), Bacillus cereus (SBT8)) and Gram-negative (Escherichia coli) were used to analyse antimicrobial activity of the plant extracts. The antimicrobial effects of the extracts were given in Table 1. The inhibition zone produced by the extracts and the standards on various bacterial strains was between $8 \mathrm{~mm}$ and $40 \mathrm{~mm}$. All extracts tested had antimicrobial activity with zones of inhibition of 8 to $25 \mathrm{~mm}$. However, Rosemary plant extract had very good inhibition zone for all bacteria tested. It had even better antimicrobial activity than AMP antibiotic against Bacillus cereus (SBT8) with zone diameter of $21 \mathrm{~mm}$.

\section{Discussion and Conclusions}

In the present study, antioxidant activities of Datura stramonium leaf, seed, Verbascum Thapsus, Rosmarinus officinalis and Thymus vulgaris plants grown in the Shar mountains of Kosovo were determined by using DPPH radical scavenging activity, ferrous ion chelating activity and reducing power methods. The results showed that all the extracts tested had very high antioxidant properties while thyme had the highest antioxidant activity among others. Our results were similar to literature work. Accordingly Amarowicz et. Al. investigated -antioxidant activity and DPPH free radical-scavenging ability of ethanolic extracts of thyme, oregano and marjoram and they showed that they had good antioxidant properties similar to our results [14].

The plant extracts from Kosovo region were also investigated to evaluate their antimicrobial effects against Staphylococcus aureus, Bacillus subtilis (ATCC 6051) 
and (ATCC 6633), Bacillus cereus (SBT8) and Escherichia coli bacteria strains. Among the tested plant extracts, the rosemary methanol extract had the best inhibitory effect to the all bacteria. Our results were similar to the work done by Moreno et. al. [15]. They evaluated the methanol and water extracts of the rosemary plant in terms of their antimicrobial activities. They found that the methanol extract had more valuable antimicrobial activity than the water extract. Ozcan et al. has similarly investigated the $\beta$-glucan content, antioxidant and antimicrobial activities of Boletus edulis, Cantharellus cibarius, Craterellus cornucopioides, Hydnum repandum and Agaricus bisporus [16]. Their results were also good and close to our results.

Overall, in the present study, biological activities of Kosovo plant extracts (Datura stramonium leaf, Datura stramonium seed, Verbascum thapsus, Rosmarinus officinalis and Thymus vulgaris) were evaluated and all showed good antioxidants and antibacterial activities. While thyme showed very high antioxidant behaviour among the others, rosemary demonstrated the best antimicrobial property against all tested bacteria. Rosemary showed a better antimicrobial activity on Bacillus cereus (SBT8) compared to ampicillin as a known antibiotic. The results of our study may indicate that among all plant extracts tested, especially thyme and rosemary extracts may be suitable for good antioxidant and antibacterial based materials for the food and pharmaceutical industries.

\section{REFERENCES}

[1] Awaad, A.S., El-Meligy, R.M., Qenawy, S.A, Atta, A.H. and Soliman, G.A. (2011). Anti-inflammatory, antinociceptive and antipyretic effects of some desert plants. Journal of Saudi Chemical Society, 15(4), 367-373.

[2] Mehrotra, S. (2005). Role of traditional and folk herbals development of new drugs. Ethanot, 17: $104-111$.

[3] Rates, S. (2001). Plants as source of drugs. Toxicon. 39, 603-613.
[4] Halliwell, B. (1997). Nutrition Reviews, 55, 44-52.

[5] Sen, C.K., Packer, L., Hanninen, O. (2000), Handbook of oxidants and antioxidants in exercise, Part 1, Elsevier Science B.V. Amsterdam.

[6] Cao, G. ve Prior, R.I. (1999). The Measurement of Oxygen Radical Absorbance Capacity in Biological Samples. Methods in Enzymology, 299, 50-62.

[7] Sagdic, O., Kuscu, A., Ozcan, M. ve Ozcelik, S. (2002). Effects of Turkish spice extracts at various concentrations on the growth E. Coli O157:H7. Food Microbiology, 19, 473-480.

[8] Srivastava, J. Chandra, H. Nautiyal, A.R. and Kalra, S.J.S. (2013). Antimicrobial resistance (AMR) and plant-derived antimicrobials (PDAms) as an alternative drug line to control infections. Biotech, 4.451-60.

[9] Blois, M.S. (1958). Antioxidant determinations by the use of stable free radical. Nature, 181, 1199-1200.

[10] Magaldi S., Mata-Essayag S., Hartung de Capriles C. (2004). Well diffusion for antifungal susceptibility testing. The International Journal of Infectious Diseases. 8, 39-45

[11] Sanchez-Moreno, C. (2002). Review: Methods Used to Evaluate the Free Radical Scavenging Activity in Foods and Biological Systems. Food Science and Technology International, 8(3), 121-137.

[12] Jovanovic, S.V., Steenken, S., Simic, M.G., Hara, Y. (1998). In Flavonoids in Health and Disease, C. Rice-Evans, L. Packer (Eds), Marcel Dekker, New York, 137-161.

[13] Oyaizu M. (1986). Studies on product of browning reaction prepared from glucoseamine. Japanese Journal of Nutrition. $44,307-15$.

[14] Amarowicz, R., Zegarska, Z., Rafalowski, R., Pegg, R.B., Karamac, M., Kosinska, A. (2009). Antioxidant activity and free radical-scavenging capacity of ethanolic extracts of thyme, oregano and marjoram. European Journal of Lipid Science and Technology. 111, 1111-1117.

[15] Moreno, S. Scheyer, T., Romano, C.S., Vojn2018ov, A.A. (2006). Antioxidant and antimicrobial activities of rosemary extracts linked to their polyphenol composition. Free Radical Research. 40 (2), 223-231.

[16] Ozcan, O., Ertan, F. (2018). Beta-glucan Content, Antioxidant and Antimicrobial Activities of Some Edible Mushroom Species. Food Science and Technology. 6(2), 47 -55 . 